\title{
Speckle Reduction in Near-field Image of Multimode Fiber with a Piezoelectric Transducer
}

\author{
Woosung $\mathrm{Ha}$, Sejin Lee, and Kyunghwan Oh* \\ Institute of Physics and Applied Physics, Yonsei University, 262 Seongsanno, Seodaemun-gu, Seoul \\ 120-749, Korea \\ Yongmin Jung \\ Optoelectronic Research Centre, University of Southampton, Highfield, Southampton, SO17 1BJ, \\ United Kingdom \\ Jun Ki Kim \\ Fraunhofer Institute for Applied Optics and Precision Engineering, Albert-Einstein Str. 7, 07745, \\ Jena, Germany
}

(Received August 1, 2008 : revised September 10, 2008 : accepted September 12, 2008)

\begin{abstract}
We propose and experimentally demonstrate an effective method to reduce near-field speckle noise at the output of a $50 \mu \mathrm{m}$ graded index multimode fiber using a short cylindrical piezoelectric transducer (PZT) vibrating in the radial direction. The fiber was coiled as tightly as possible around the mandrel of the PZT and a periodic stretching effect was caused by the radial oscillations of the actuator. The output of the optical fiber using the He-Ne laser source was intensively observed by a CCD camera. By counting all the pixels corresponding to relative intensity graded into 256 levels in the selected area and by calculating standard deviation and mean value of the intensity, we could measure the speckle contrast and vibration effect quantitatively with reduction ratio of pixels and line profile of the illuminated region. It was clearly observed that the characteristics of the speckle pattern in the vibration-on state were significantly improved over that of the vibration-off state due to time-averaged smoothing.
\end{abstract}

Keywords : speckle reduction; multimode fiber; piezoelectric transducer; radial vibration, timeaveraged smoothing

OCIS codes : (060.2310) Fiber optics; (100.1160) Analog optical image processing; (110.1650) Coherence Imaging; (110.2350) Fiber optics imaging; (110.6150) Speckle imaging

\section{INTRODUCTION}

When a coherent light is guided along a multimode fiber (MMF), a speckle pattern is formed at the waveguide output. These speckles are caused by interference between guided modes traveling within the fiber that result in degrading the laser beam quality in applications of laser marking and projection display as well as in medical surgeries. In fact, the fiber speckle patterns are extremely sensitive to external perturbations, for example, fiber stresses and wavelength changes of the source [1]. Therefore, previously various attempts have

*Corresponding author: koh@yonsei.ac.kr been made to overcome the speckle problem in guided optics. The simplest way to reduce the speckle contrast was to increase the number of high-order modes excited within a fiber by increasing the fiber length, the launched $\mathrm{NA}$, or the number of bends [2]. While this is effective for smoothing speckle in conventional step index fibers, it would not be constructive for some specialty fiber, as higher-order modes exhibit large losses within such fibers.

Other published techniques rely on creating some variation in the speckle pattern and averaging it over time. This may be done by vibrating the fiber by using a rotating diffractive optical element $[3,4]$, or even a 
varying magnetic field [5]. The speckle patterns could be reduced by the phase modulation when the waveguide is vibrated [6]. Based on this effect in addition to fiber bending, Kajenski et al. found that the speckle patterns can be affected by mode coupling and phase modulation simultaneously and that modulation of speckle is dominated by the phase modulation when a highly coherent source is used [7]. Although vibrating the fiber has been found to be a fruitful method, Kajenski's model had a weak point in that their system was not compatible with integrated optical circuits.

In this paper, we introduce an efficient compact-size system to reduce near-field speckle noises in $50 \mu \mathrm{m}$ graded index MMF using a short cylindrical piezoelectric transducer (PZT) which has been widely utilized for the phase modulation in tomography [8]. We examined the optimized condition minimizing speckles by adjusting different driving frequencies of the modulation, and the effects at the resonance were observed quantitatively with the He-Ne laser source.

\section{EXPERIMENTAL RESULTS}

The schematic setup of the measurement system is shown in Fig. 1. In this experiment, a $635 \mathrm{~nm} \mathrm{He}-\mathrm{Ne}$ laser which is highly coherent was incident on the $50 \mu \mathrm{m}$ standard graded index MMF, and the light was passed through the piezo-fiber assembly and guided into the

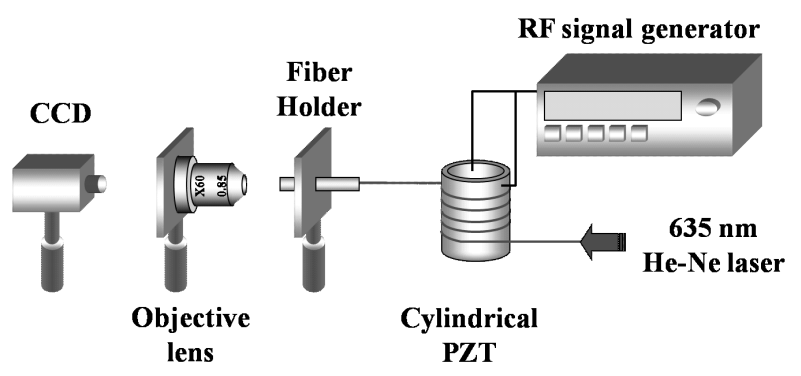

FIG. 1. Schematics of the experimental setup.

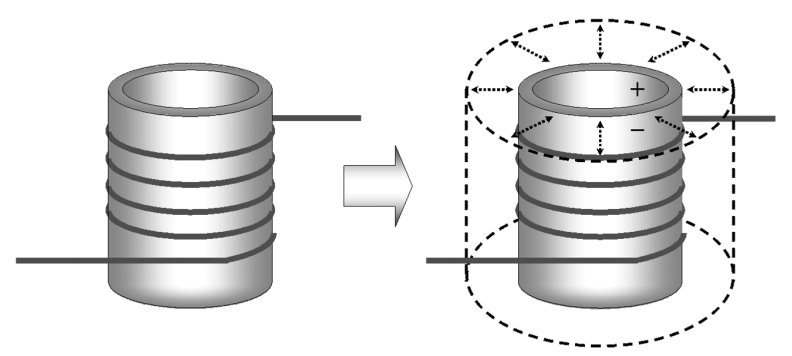

FIG. 2. Piezo-fiber assembly vibrating in radial direction. 2 meter-long graded index MMF of $50 \mu \mathrm{m}$ core size was coiled 20 rounds around the mandrel of the actuator as tightly as possible.
CCD directly through a $\times 60$ objective lens (NA 0.85 ). Throughout the experimental process, the power of the laser source was kept constant and external vibrations were minimized on a floated optical table because the beam pattern is very sensitive to perturbation along the optical path. Actual images of vibration-off/on states were captured by the CCD camera and quantitative analysis was performed by counting the number of pixels of 256-level intensity in the selected area with a National Instrument IMAQ Vision Builder.

The mechanism of the piezo-fiber assembly is shown in Fig. 2. The cylindrical PZT used in this experiment was an EC-65 having radial displacement less than 0.4 $\mu \mathrm{m} / \mathrm{V}$ and produced by EDO Corporation. This product has an outer diameter of $38 \mathrm{~mm}$ and $2 \mathrm{~mm}$ thickness and is made of lead zirconate titanate. With this actuator, MMF is coiled 20 rounds around the axis of the transducer as tightly as possible, and bonded by epoxy. Note that if the number of coils was more than 20 there was significant loss of light during guiding, and a fundamental mode only at the output. By winding the fiber with a sinusoidal driving signal, bending effect and phase modulation occur simultaneously and speckle patterns can be time-averaged. To obtain effective time-averaged smoothing of the multiple spatial modes in the fiber, the phase of each mode should be varied by more than $2 \pi$ on time scales faster than the response time of the measurement system [9].

The problem is to define what factor is the standard of speckle strength. Physically, speckle of the fiber output means dark pixels in the circular region. Strong speckle showing high contrast, therefore, will result in large deviation of intensity in the selected area. On the other hand, weak speckle, that is to say uniform output beam, will have small deviation. Hence we can think the standard deviation of intensity as the uniformity of the beam. If the amplitude of radial oscillation increases, the phase modulation effect will be increased and will show reduced speckle pattern. Therefore the time-averaged smoothing effect will maximize the reduction of speckle at the resonant driven frequency. The lowest resonant frequency in radial vibration can be evaluated according to the reference [10], and the PZT, thus, will have the frequency near $24 \mathrm{kHz}$. Therefore the measurement was considered in the frequency range from 22 to $26 \mathrm{kHz}$ to find the lowest resonant frequency with 10 volt peak to peak. By taking all the images at the modulation frequency range with increment of $0.01 \mathrm{kHz}$ controlled by the function generator (Agilent 33120A), we obtained a total of 401 images with all the other experimental conditions fixed. Analysis for each picture was performed over the exact same spatial region. For the circular area of 18,980 pixels, Vision Builder counted the number of pixels and calculated the standard deviation and the mean value. The resultant plot of the standard deviation and mean value of intensity are shown in Fig. 3. Fig. 3-(a) shows 


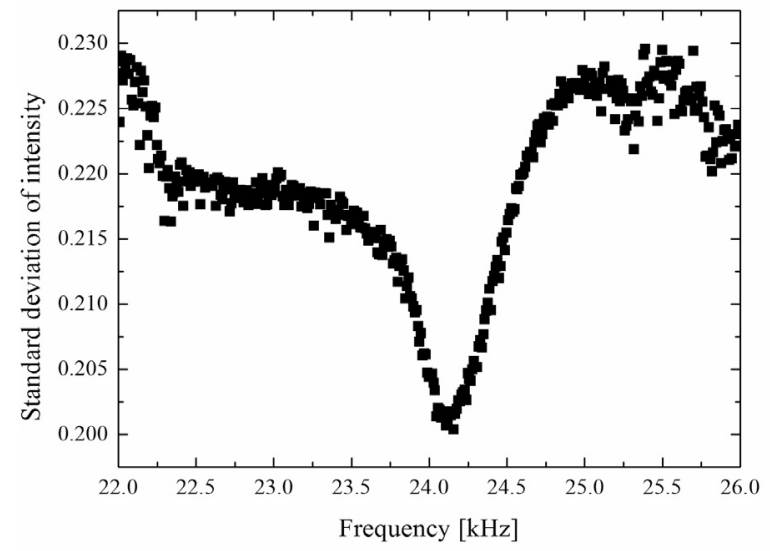

(a)

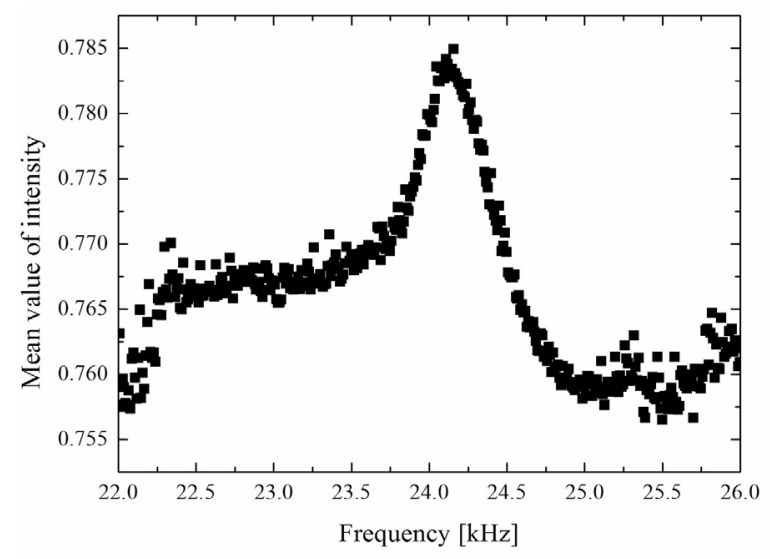

(b)

FIG. 3. (a) Standard deviation of intensity as a function of frequency between 22 and $26 \mathrm{kHz}$. (b) Mean value of intensity as a function of frequency for the same range.

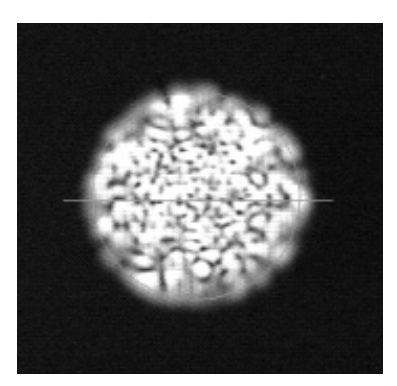

(a)

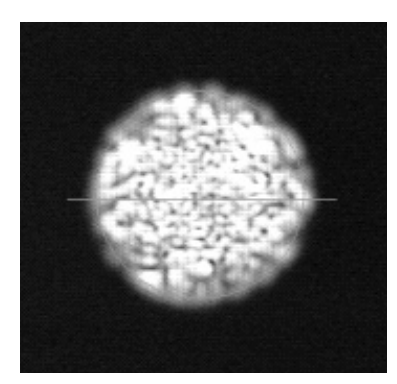

(b)
FIG. 4. Captured images through CCD camera for (a) the vibration-off state and (b) the vibration-on state at the resonant frequency of $24.16 \mathrm{kHz}$. The contrast of the image was greatly relieved for (b).

that the maximum speckle reduction occurred at 24.16 $\mathrm{kHz}$, which is the lowest resonant frequency of the PZT to radial direction. It is interesting that the shape of Fig. 3-(b) is roughly turned upside down. This phenomenon can be contributed to the removing of optical power loss due to speckle disturbance.

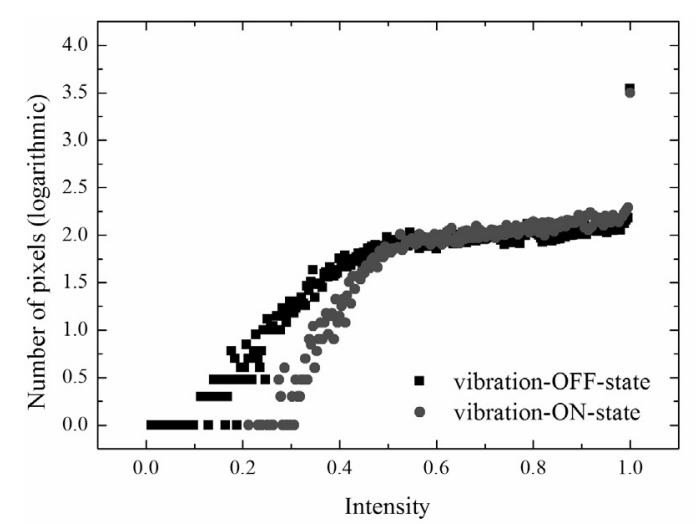

(a)

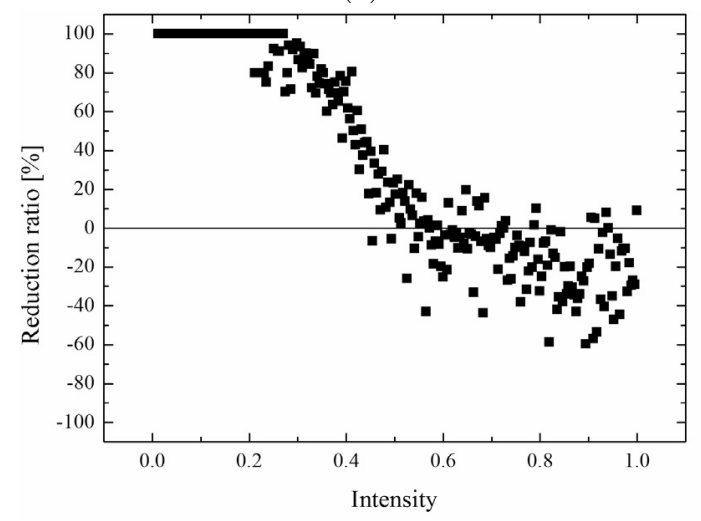

(b)

FIG. 5. (a) Logarithmic scale of the number of pixels as a function of intensity. (b) Reduction ratio for all range of intensity based on the result of (a).

In order to check the vibration effect, captured images of the vibration-off/on states at the resonant frequency of $24.16 \mathrm{kHz}$ are shown in Fig. 4 . We see speckle noise with high contrast in Fig. 4-(a), on the other hand, the speckles are reduced by the vibration, and the contrast between bright and dark region is relieved by timeaveraged smoothing in Fig. 4-(b).

Even though the effect of radial oscillation can be confirmed by the naked eye, we analyzed all the pixels in the selected area to investigate the effect quantitatively. 18,980 pixels in the green circular line of Fig. 4 were analyzed and note that exactly same location was also observed to obtain the data of Fig. 3. By grading that pure black color has an intensity of zero and that white color has an intensity of 255 , the intensity of the output beam can be identified among 256 levels. After acquiring all data, the intensity was rescaled from zero to unity. The result of this quantitative comparison between vibration-off and -on states is plotted in Fig. 5-(a) by counting the number of pixels for all 256-graded intensity. Based on this data, the reduction ratio could be achieved by dividing the difference of the number of pixels corresponding to each intensity between two states into the number of pixels of the vibration-off state. Therefore 


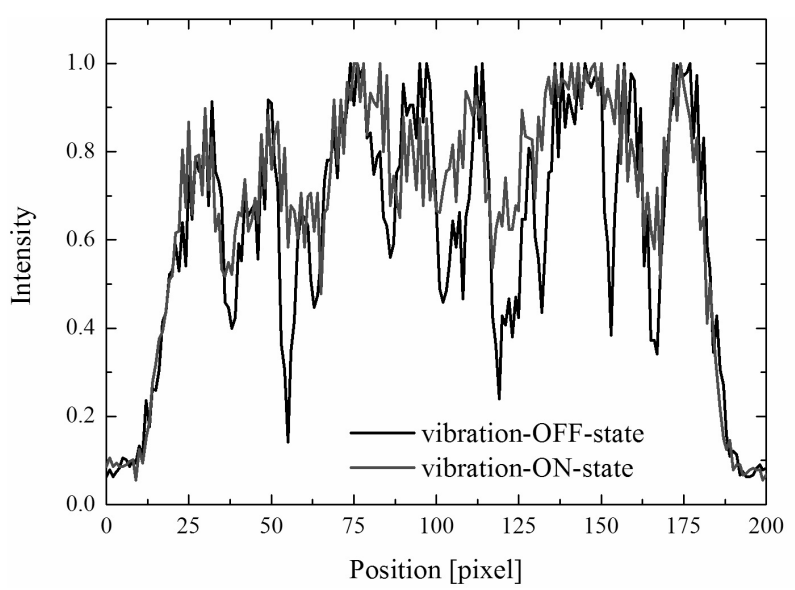

FIG. 6. Line profile of the output beam for the remarked as green line in Fig. 4 for both normal and vibrating states. Disordered line somewhat modified flatly at the illuminated region.

the reduction ratio means how many pixels corresponding to the intensity vanished or increased. The result of this calculation is shown in Fig. 5-(b). An apparent difference is shown in the figure that dark pixels were removed and bright pixels were increased by the oscillation of the actuator; the dark pixels weaker than intensity of 0.3 were almost all removed and the ratio decreases until 0.5. The numbers of pixels are commonly conserved or slightly increase in the range from 0.5 to 1 .

We easily guess that the line profile of the fiber output at the normal state will have a jagged form. The goal of this study is to make such an unstable beam profile more uniform. According to above result of the experiment, it is expected that the piezo-fiber assembly would mitigate this unstable profile into pulse shape or Gaussian type. The measurement was taken at the line in Fig. 4 , and the result is shown in Fig. 6. You can see that the line profile somewhat changed as a form with reduced noise, especially at the position near 50, 125, and 150 where the homogeneity of the beam was greatly improved and therefore the output image became more flat-topped.

\section{DISCUSSION AND CONCLUSION}

We have experimentally studied the effective speckle noise reducing method in $50 \mu \mathrm{m}$ graded index MMF using phase modulation and mode coupling with a piezoelectric actuator oscillating in the radial direction. Speckle noise has generally been used in optical signal processing and there have been experiments to measure only the spatial coherence function of the laser beam qualitatively by comparing experimental images with theory [11]. However, in this paper, quantitative analysis of speckle reduction was investigated for the first time by digital images using a CCD camera with the National Instrument IMAQ Vision Builder. The analysis was restricted to near-field region to find a potential as a beam homogenizer in the area of fiber laser marking applications. It was shown that the proposed method can reduce speckles significantly especially for the pixels having extremely low intensity due to phase modulation by radial oscillation of actuator.

Although we could not achieve the perfectly flat beam type, the authors found ample potential of the beam homogenizer which can be developed as an integrated circuit owing to its considerably reduced mechanical size of operation. Because time-averaged smoothing depends on the performance of the piezoelectric actuator, the reduction ratio and the homogeneity of the beam will be surely improved with larger oscillation amplitude by adopting higher performance of piezoelectric material or by using an RF signal amplifier to raise the driven voltage.

Further investigations on far-field speckle patterns are being pursued by the authors as well as for other types of optical fibers such as a hard polymer cladding optical fiber. We expect that the proposed speckle reduction technology would find viable applications in fiber laser marking systems.

\section{ACKNOWLEDGMENT}

This work was supported in part by the KOSEF (Program Nos. ROA-2008-000-20054-0, R01-2006-00011277-0, and R15-2004-024-00000-0), the KICOS (Program Nos. 2007-8-0536 and 2007-8-1864), the ITEP (Program No. 2007-8-2074), and the Brain Korea 21 Project of the KRF.

\section{REFERENCES}

[1] K.-I. Sato and K. Asatani, "Speckle Noise Reduction in Fiber Optic Analog Video Transmission Using Semiconductor Laser Diodes," IEEE Trans. Commun., vol. 29, no. 7, pp. 1017-1024, 1981.

[2] D. J. Anderson, R. D. Morgan, D. R. McCluskey, J. D. C. Jones, W. J. Easson, and C. A. Greated, “An optical fibre delivery system for pulsed laser particle image velocimetry illumination," Meas. Sci. Technol., vol. 6, pp. 809-1814, 1995.

[3] L. Wang, T. Tschudi, T. Halldórsson, and P. R. Pétursson, "Speckle reduction in laser projection systems by diffractive optical elements," Appl. Opt., vol. 37, no. 10, pp. 1770-1775, 1998.

[4] J. I. Trisnadi, "Hadamard speckle contrast reduction," Opt. Lett., vol. 29, no. 1, pp. 11-13, 2004.

[5] L. I. Ardasheva, N. D. Kundikova, M. O. Sadykova, N. R. Sadykov, and V. E. Chernyakov, "Speckle mode rotation in a few mode optical fiber in a longitudinal magnetic field," Opt. Spectrosc., vol. 95, pp. 645-651, 2003. 
[6] S. A. Kingsley and D. E. N. Davies, "Multimode optical fibre phase modulators and discriminators: I-Theory," Electron. Lett., vol. 14, no. 11, pp. 322-325, 1978.

[7] P. J. Kajenski, P. L. Fuhr, and D. R. Huston, "Mode Coupling and Phase Modulation in Vibrating Waveguides," IEEE J. Lightwave Technol., vol. 10, no. 9, pp. 1297-1301, 1992.

[8] W. J. Choi, J. Na, S. Y. Ryu, B. H. Lee, and D.-S. Ko, "Realization of 3-D Topographic and Tomograpic Images with Ultrahigh-resolution Full-field Optical Coherence Tomography," J. Opt. Soc. Korea, vol. 11, no. 1, pp. 18-25, 2007.
[9] A. P. Povilus, S. E. Olson, R. R. Mhaskar, B.-K. Teo, J. R. Guest, and G. Raithel, "Time averaging of multimode optical fiber output for a magneto-optical trap," J. Opt. Soc. Am. B, vol. 22, no. 2, pp. 311-314, 2005.

[10] J. O. Kim, K. K. Hwang, and H. G. Jeong, "Radial vibration characteristics of piezoelectric cylindrical transducers," J. Sound Vib., vol. 276, pp. 1135-1144, 2004.

[11] C.-H. Lee, Y. Kang, Y. Sung, and J. Noh, "Measurement of Spatial Coherence Function of Laser Beam by using a Sagnac Interferometer," J. Opt. Soc. Korea, vol. 11, no. 2, pp. 71-75, 2007. 